\title{
Partially defined $\sigma$-derivations on semisimple Banach algebras
}

\author{
by \\ Tsiu-Kwen Lee (Taipei) and Cheng-Kai Liu (Changhua)
}

\begin{abstract}
Let $A$ be a semisimple Banach algebra with a linear automorphism $\sigma$ and let $\delta: I \rightarrow A$ be a $\sigma$-derivation, where $I$ is an ideal of $A$. Then $\Phi(\delta)(I \cap \sigma(I))=0$, where $\Phi(\delta)$ is the separating space of $\delta$. As a consequence, if $I$ is an essential ideal then the $\sigma$-derivation $\delta$ is closable. In a prime $C^{*}$-algebra, we show that every $\sigma$-derivation defined on a nonzero ideal is continuous. Finally, any linear map on a prime semisimple Banach algebra with nontrivial idempotents is continuous if it satisfies the $\sigma$-derivation expansion formula on zero products.
\end{abstract}

1. Results. Throughout the paper, $A$ is always a unital Banach algebra over the complex field $\mathbb{C}$ and $\sigma$ is a linear endomorphism of $A$. Let $1_{A}$ denote the identity automorphism of $A$. By a $\sigma$-derivation of $A$ we mean a linear map $\delta: A \rightarrow A$ such that $\delta(x y)=\sigma(x) \delta(y)+\delta(x) y$ for all $x, y \in A$. Clearly, the map $\sigma-1_{A}$ is a $\sigma$-derivation and $1_{A}$-derivations are just ordinary derivations. Thus the concept of $\sigma$-derivations can be regarded as a generalization of both derivations and endomorphisms. Let $I$ be a nonzero ideal of $A$. A linear map $\delta: I \rightarrow A$ is called a $\sigma$-derivation defined on $I$ if $\delta(x y)=\sigma(x) \delta(y)+\delta(x) y$ for all $x, y \in I$. An ideal $I$ of $A$ is called essential if $I$ has nontrivial intersection with any nonzero ideal of $A$. For a semisimple algebra $A$, this is equivalent to saying that $a I=0$ where $a \in A$ implies $a=0$. A $\sigma$-derivation $\delta: I \rightarrow A$ is called essentially defined on an ideal $I$ if $I$ is an essential ideal of $A$.

Kaplansky conjectured that every derivation on a $C^{*}$-algebra is continuous [16] and that every derivation on a semisimple Banach algebra is continuous [17]. Sakai confirmed Kaplansky's conjecture for $C^{*}$-algebras in [22]. The second conjecture was confirmed by Johnson and Sinclair in [15].

2000 Mathematics Subject Classification: 46H40, 47B47, 46H15.

Key words and phrases: Banach algebra, prime $C^{*}$-algebra, $\sigma$-derivation, closability, continuity.

C.-K. Liu is the corresponding author. T.-K. Lee is a member of the Mathematics Division, NCTS at Taipei. 
Many related results have been obtained in the literature (see, for instance, $[3,4,9,15,21,24,26,27])$. In [27] Villena proved that every derivation defined on an essential ideal of a semisimple Banach algebra is automatically closable. As an application, he showed that every derivation defined on a nonzero ideal of a prime $C^{*}$-algebra is continuous. Recently, several results concerning $\sigma$-derivations of Banach algebras have been studied (see $[1,5,6$, $9,12,13,19,21])$. Brešar and Villena [9] proved that if $A$ is a semisimple Banach algebra and $\sigma$ is a linear automorphism of $A$, then every $\sigma$-derivation on $A$ is automatically continuous. In this paper, instead of essential ideals, we investigate partially defined $\sigma$-derivations on any nonzero ideal.

To state our results precisely, we recall the definition of separating spaces. Let $X$ and $Y$ be normed spaces over the complex field $\mathbb{C}$ and let $T: X \rightarrow Y$ be a linear map. The separating space $\Phi(T)$ of $T$ is defined as follows:

$$
\begin{aligned}
& \Phi(T)=\left\{y \in Y \mid \text { there exists a sequence }\left(x_{n}\right) \text { in } X\right. \text { with } \\
& \left.\qquad \lim _{n \rightarrow \infty} x_{n}=0 \text { and } \lim _{n \rightarrow \infty} T\left(x_{n}\right)=y\right\} .
\end{aligned}
$$

Clearly, $\Phi(T)$ is a subspace of $Y$. We say that $T$ is closable if $\Phi(T)=\{0\}$. For Banach spaces $X$ and $Y$, the closed graph theorem asserts that $T$ is continuous if and only if it is closable. We are now ready to state the main theorem of the paper.

TheOREM 1.1. Let $A$ be a semisimple Banach algebra with a linear epimorphism $\sigma$ and let $\delta: I \rightarrow A$ be a $\sigma$-derivation, where $I$ is an ideal of $A$. Then $\Phi(\delta)(I \cap \sigma(I))=0$. As a consequence, every essentially defined $\sigma$ derivation on $A$ is closable if $\sigma$ is a linear automorphism of $A$.

As applications of Theorem 1.1, we have the following two results.

Corollary 1.2. Let $A$ be a semisimple Banach algebra with a linear epimorphism $\sigma$. Then every $\sigma$-derivation on $A$ is continuous.

Corollary 1.3. Let $A$ be a prime $C^{*}$-algebra with a linear automorphism $\sigma$ and let $I$ be a nonzero ideal of $A$. Then every $\sigma$-derivation $\delta: I \rightarrow A$ is continuous.

Recently, there have been much work concerning maps preserving zero products in the literature (see $[8,10,11,14,18,28]$ ). Applying Theorem 1.1 we obtain the continuity of linear maps which satisfy the $\sigma$-derivation expansion formula on zero products.

THEOREM 1.4. Let $A$ be a prime semisimple Banach algebra with nontrivial idempotents and let $\sigma$ be a linear automorphism of $A$. Suppose that $\delta: A \rightarrow A$ is a linear map such that $\sigma(x) \delta(y)+\delta(x) y=0$ for all $x, y \in A$ with $x y=0$. Then $\delta$ is continuous. 
2. Preliminaries. We fix some notation and terminology. Let $A$ be a semisimple Banach algebra. Recall that $\operatorname{soc}(A)$, the socle of $A$, is defined as the sum of all minimal left ideals of $A$. Therefore, each element in $\operatorname{soc}(A)$ lies in a sum of finitely many minimal left ideals of $A$. The $\operatorname{socle} \operatorname{soc}(A)$ also coincides with the sum of all minimal right ideals of $A$. An element $a \in A$ is said to be of rank one if $a A$ is a minimal right ideal of $A$. This is equivalent to saying that $A a$ is a minimal left ideal of $A$. Moreover, $a \in \operatorname{soc}(A)$ has rank one if and only if $a A a=\mathbb{C} a$. Thus, if $a \in \operatorname{soc}(A)$ has rank one, then $A a$ is a minimal left ideal and $a A$ is a minimal right ideal of $A$. Let $P$ be a primitive ideal of $A$ and let $\pi$ be a continuous irreducible representation of $A$ with $\operatorname{ker} \pi=P$. Then $a+P \in \operatorname{soc}(A / P)$ if and only if $\pi(a)$ is a finite rank operator (see [7] for details).

We begin with several lemmas.

Lemma 2.1. Let $A$ be a semisimple Banach algebra. If $a, b \in \operatorname{soc}(A)$ then $a A b$ is finite-dimensional over $\mathbb{C}$.

Proof. Obviously, we may assume $a A b \neq 0$. Suppose first that both $a$ and $b$ have rank one. Thus $a A a=\mathbb{C} a$ and $A b$ is a minimal left ideal of $A$. Choose $x \in A$ such that $a x b \neq 0$. Then $A b=A a x b$ by minimality of $A b$. Thus $a A b=a A a x b=(a A a) x b=\mathbb{C} a x b$, implying $\operatorname{dim}_{\mathbb{C}} a A b=1$, as desired.

Let $a, b \in \operatorname{soc}(A)$. There are finitely many elements $a_{1}, \ldots, a_{m}, b_{1}, \ldots, b_{n}$ in $A$ of rank one such that $a=\sum_{i=1}^{m} a_{i}$ and $b=\sum_{j=1}^{n} b_{j}$. Note that $\operatorname{dim}_{\mathbb{C}} a_{i} A b_{j} \leq 1$ for all $i, j$. Then $a A b \subseteq \sum_{i=1}^{m} \sum_{j=1}^{n} a_{i} A b_{j}$, implying $\operatorname{dim}_{\mathbb{C}} a A b \leq m n$. This proves the lemma.

We also need the gliding hump argument due to Thomas [25, Proposition 1.3] and Johnson and Sinclair's lemma [25, Lemma 1.5], which are essential to our proofs.

Lemma 2.2 (Gliding hump argument). Let $X, Y$ and $\left\{Y_{i}\right\}_{i=1}^{\infty}$ be Banach spaces. Let $\left\{T_{i}\right\}_{i=1}^{\infty}$ be a sequence of continuous linear operators from $X$ into itself and let $\left\{U_{i}\right\}_{i=1}^{\infty}$ be a sequence of continuous linear operators, where each $U_{i}: Y \rightarrow Y_{i}$. If $S$ is a linear operator from $X$ to $Y$ such that $U_{n} S T_{1} T_{2} \cdots T_{m}$ is continuous for $m>n$, then $U_{n} S T_{1} T_{2} \cdots T_{n}$ is continuous for sufficiently large $n$.

Lemma 2.3 (Johnson and Sinclair). Let $A$ be a Banach algebra and let $\pi$ be a continuous irreducible representation of $A$ on an infinite-dimensional normed complex linear space $X$. Let $\left\{x_{i}\right\}_{i=0}^{\infty}$ be a linearly independent subset of $X$. Then there exists a sequence $\left\{a_{i}\right\}_{i=1}^{\infty}$ in $A$ such that $\pi\left(a_{m} \cdots a_{1}\right) x_{n}=0$ for all $m>n \geq 0$ and $\left\{\pi\left(a_{n} \cdots a_{1}\right) x_{l}\right\}_{l=n}^{\infty}$ is a linearly independent subset of $X$ for all $n \geq 1$.

Lemma 2.4. Let $A$ be a Banach algebra and let $\sigma$ be a continuous epimorphism of $A$. Let $\pi_{i}$ be a continuous irreducible representation of $A$ on 
the Banach space $X_{i}$ with $\operatorname{ker} \pi_{i}=P_{i}$ for $i=1,2, \ldots$ Suppose that $\delta: I \rightarrow A$ is a $\sigma$-derivation, where $I$ is a nonzero ideal of $A$. Suppose that there exist a sequence $\left\{c_{i}\right\}_{i=1}^{\infty}$ in $I$, a sequence $\left\{b_{i}\right\}_{i=0}^{\infty}$ in $A$ with $b_{0} \in I$, and a sequence $\left\{x_{i}\right\}_{i=1}^{\infty}$, where $x_{i} \in X_{i}$ for $i \geq 1$, such that

- $\sigma\left(c_{n}\right) \notin P_{n}$ for all $n \geq 1$,

- $\pi_{n}\left(b_{n} \cdots b_{1} b_{0}\right) x_{n} \neq 0$

- $\pi_{n}\left(b_{m} \cdots b_{1} b_{0}\right) x_{n}=0$ for all $m>n \geq 1$.

Then $\Phi(\delta) \subseteq P_{k}$ for some $k \geq 1$.

Proof. Let $U_{n}: A \rightarrow X_{n}, T_{n}: A \rightarrow A$ and $R_{b_{0}}: A \rightarrow I$ be continuous linear operators given by

$$
U_{n}(a)=\pi_{n}\left(\sigma\left(c_{n}\right) a\right) x_{n}, \quad T_{n}(a)=a b_{n} \quad \text { and } \quad R_{b_{0}}(a)=a b_{0}
$$

for $a \in A$ and for $n \geq 1$. Notice that $\delta R_{b_{0}}$ is a linear operator from $A$ into itself. Then if $m>n$, we have

$$
\begin{aligned}
U_{n}( & \left.\delta R_{b_{0}}\right) T_{1} \cdots T_{m}(a) \\
& =\pi_{n}\left(\sigma\left(c_{n}\right) \delta\left(a b_{m} \cdots b_{1} b_{0}\right)\right) x_{n} \\
& =\pi_{n}\left(\delta\left(c_{n} a b_{m} \cdots b_{1} b_{0}\right)-\delta\left(c_{n}\right) a b_{m} \cdots b_{1} b_{0}\right) x_{n} \\
& =\pi_{n}\left(\sigma\left(c_{n} a\right) \delta\left(b_{m} \cdots b_{1} b_{0}\right)+\delta\left(c_{n} a\right) b_{m} \cdots b_{1} b_{0}-\delta\left(c_{n}\right) a b_{m} \cdots b_{1} b_{0}\right) x_{n} \\
& =\pi_{n}\left(\sigma\left(c_{n}\right) \sigma(a) \delta\left(b_{m} \cdots b_{1} b_{0}\right)\right) x_{n} .
\end{aligned}
$$

Thus $U_{n}\left(\delta R_{b_{0}}\right) T_{1} \cdots T_{m}$ is continuous for all $m>n$. By Lemma 2.2, there exists an integer $n \geq 1$ such that $U_{n}\left(\delta R_{b_{0}}\right) T_{1} \cdots T_{n}$ is continuous. Let $b, c \in I$ and let $\left\{a_{k}\right\}_{k=1}^{\infty}$ be a sequence in $I$ with $\lim _{k \rightarrow \infty} a_{k}=0$ and $\lim _{k \rightarrow \infty} \delta\left(a_{k}\right)=$ $a \in \Phi(\delta)$. Then $\lim _{k \rightarrow \infty} c a_{k} b=0$. Since

$$
\begin{aligned}
U_{n}\left(\delta R_{b_{0}}\right) T_{1} \cdots & T_{n}\left(c a_{k} b\right) \\
= & \pi_{n}\left(\sigma\left(c_{n}\right) \delta\left(c a_{k} b b_{n} \cdots b_{1} b_{0}\right)\right) x_{n} \\
= & \pi_{n}\left(\sigma\left(c_{n}\right)\right) \pi_{n}\left(\sigma\left(c a_{k}\right) \delta\left(b b_{n} \cdots b_{1} b_{0}\right)\right. \\
& \left.+\sigma(c) \delta\left(a_{k}\right) b b_{n} \cdots b_{1} b_{0}+\delta(c) a_{k} b b_{n} \cdots b_{1} b_{0}\right) x_{n} \\
= & \pi_{n}\left(\sigma\left(c_{n}\right)\right) \pi_{n}(\sigma(c)) \pi_{n}\left(\sigma\left(a_{k}\right)\right) \pi_{n}\left(\delta\left(b b_{n} \cdots b_{1} b_{0}\right)\right) x_{n} \\
& +\pi_{n}\left(\sigma\left(c_{n}\right)\right) \pi_{n}(\sigma(c)) \pi_{n}\left(\delta\left(a_{k}\right)\right) \pi_{n}(b) \pi_{n}\left(b_{n} \cdots b_{1} b_{0}\right) x_{n} \\
& +\pi_{n}\left(\sigma\left(c_{n}\right)\right) \pi_{n}(\delta(c)) \pi_{n}\left(a_{k}\right) \pi_{n}(b) \pi_{n}\left(b_{n} \cdots b_{1} b_{0}\right) x_{n},
\end{aligned}
$$

it is easy to see that

$$
\begin{aligned}
0 & =\lim _{k \rightarrow \infty} U_{n} \delta R_{b_{0}} T_{1} \cdots T_{n}\left(c a_{k} b\right) \\
& =\pi_{n}\left(\sigma\left(c_{n}\right)\right) \pi_{n}(\sigma(c)) \pi_{n}(a) \pi_{n}(b) \pi_{n}\left(b_{n} \cdots b_{1} b_{0}\right) x_{n} .
\end{aligned}
$$


Thus

$$
\pi_{n}\left(\sigma\left(c_{n}\right)\right) \pi_{n}(\sigma(I)) \pi_{n}(a) \pi_{n}(I) \pi_{n}\left(b_{n} \cdots b_{1} b_{0}\right) x_{n}=0 .
$$

Recall that $\sigma\left(c_{n}\right) \notin P_{n}$ and $b_{0} \notin P_{n}$. So $\sigma(I) \nsubseteq P_{n}$ and $I \nsubseteq P_{n}$. In particular, we have $\pi_{n}(I) \pi_{n}\left(b_{n} \cdots b_{1} b_{0}\right) x_{n}=X_{n}$. It follows from (1) that $\sigma\left(c_{n}\right) \sigma(I) a \subseteq$ $\operatorname{ker} \pi_{n}=P_{n}$. Recall that $\sigma(I)$ is an ideal of $A$ and $\sigma(I) \nsubseteq P_{n}$. This implies $a \in P_{n}$, as desired.

Lemma 2.5. Let $A$ be a Banach algebra and let $\sigma$ be a continuous epimorphism of $A$. Suppose that $\delta: I \rightarrow A$ is a $\sigma$-derivation, where $I$ is a nonzero ideal of $A$. If $P$ is a primitive ideal of $A$ satisfying $I \nsubseteq P$ and $\sigma(I) \nsubseteq P$, then either $\Phi(\delta) \subseteq P$ or $(I+P) / P=\operatorname{soc}(A / P)$.

Proof. By assumption, there exists $c \in I$ such that $\sigma(c) \notin P$. Suppose that $(I+P) / P \neq \operatorname{soc}(A / P)$. Let $\pi$ be a continuous irreducible representation of $A$ on an infinite-dimensional Banach space $X$ with $\operatorname{ker} \pi=P$. Then $\operatorname{dim}_{\mathbb{C}} \pi\left(b_{0}\right) X=\infty$ for some $b_{0} \in I$. Hence $\left\{\pi\left(b_{0}\right) x_{i}\right\}_{i=0}^{\infty}$ is a linearly independent subset of $X$ for some $x_{i} \in X, i \geq 0$. By Lemma 2.3, there exists a sequence $\left\{b_{i}\right\}_{i=1}^{\infty}$ in $A$ such that $\pi\left(b_{n} \cdots b_{1}\right) \pi\left(b_{0}\right) x_{n} \neq 0$ and $\pi\left(b_{m} \cdots b_{1}\right) \pi\left(b_{0}\right) x_{n}=0$ for all $m>n$. Now we let $c_{i}=c, \pi_{i}=\pi$ and $P_{i}=P$ for all $i \geq 1$. In view of Lemma 2.4, we obtain $\Phi(\delta) \subseteq P$, proving the lemma.

Lemma 2.6. Let $A$ be a Banach algebra, $P$ a primitive ideal of $A$ and $\sigma$ a continuous epimorphism of $A$. Suppose that $\delta: I \rightarrow A$ is a $\sigma$-derivation defined on a nonzero ideal $I$ of $A$. If there exist $c, b \in I$ such that $\sigma(c) \notin P$, $b \notin P$ and $\operatorname{dim}_{\mathbb{C}} c A b<\infty$, then $\Phi(\delta) \subseteq P$.

Proof. Since $\operatorname{dim}_{\mathbb{C}} c A b<\infty$, the map $a \in A \mapsto \delta(c a b)$ is continuous. Let $\left\{a_{n}\right\}_{n=1}^{\infty}$ be a sequence in $I, \lim _{n \rightarrow \infty} a_{n}=0$ and $\lim _{n \rightarrow \infty} \delta\left(a_{n}\right)=a \in \Phi(\delta)$. Let $x, y \in I$. Since $\delta\left(c x a_{n} y b\right)=\sigma(c x) \sigma\left(a_{n}\right) \delta(y b)+\sigma(c x) \delta\left(a_{n}\right) y b+\delta(c x) a_{n} y b$, it is easy to see that $\lim _{n \rightarrow \infty} \delta\left(c x a_{n} y b\right)=\sigma(c x) a y b=0$. This implies that $\sigma(c) \sigma(I) a I b=0$. From $\sigma(c), b \notin P$ and $\sigma(I), I \nsubseteq P$, it follows that $a \in P$. Thus $\Phi(\delta) \subseteq P$, as desired.

\section{Proofs}

Proof of Theorem 1.1. By [23, Corollary 6.12], $\sigma$ is continuous on $A$. Let $\Sigma$ denote the set of all primitive ideals of $A$. Since $A$ is semisimple, $\bigcap_{P \in \Sigma} P=0$. Set

$$
\Sigma_{N}=\{P \in \Sigma \mid I \nsubseteq P \text { and } \sigma(I) \nsubseteq P\} .
$$

For $P \in \Sigma$ and $P \notin \Sigma_{N}$, we have $I \cap \sigma(I) \subseteq P$. Thus $(I \cap \sigma(I)) \cap \bigcap_{P \in \Sigma_{N}} P=0$. Next we set $\Sigma_{I}=\left\{P \in \Sigma_{N} \mid \Phi(\delta) \nsubseteq P\right\}$. So $\Phi(\delta) \subseteq P$ if $P \in \Sigma_{N} \backslash \Sigma_{I}$. 
Suppose first that $\Sigma_{I}=\emptyset$. Then $\Phi(\delta) \subseteq \bigcap_{P \in \Sigma_{N}} P$. This implies that

$$
\Phi(\delta)(I \cap \sigma(I)) \subseteq\left(\bigcap_{P \in \Sigma_{N}} P\right) \cap(I \cap \sigma(I))=0,
$$

proving the theorem. Assume on the contrary that $\Sigma_{I} \neq \emptyset$. Let $K=$ $\bigcap_{P \in \Sigma_{N} \backslash \Sigma_{I}} P$ and $J=(I \cap \sigma(I)) \cap K$. Then $J$ is an ideal of $A$ contained in $I$ and $J \subseteq \bigcap_{P \in \Sigma \backslash \Sigma_{I}} P$.

If $J \subseteq P$ for some $P \in \Sigma_{I}$, then $I \sigma(I) K \subseteq J \subseteq P$. Since $I \nsubseteq P$ and $\sigma(I) \nsubseteq P$, we have $K \subseteq P$. Thus $\Phi(\delta) \subseteq K \subseteq P$, a contradiction. Hence we may choose $P_{0} \in \Sigma_{I}$ such that $J \nsubseteq P_{0}$. By Lemma $2.5,0 \neq\left(J+P_{0}\right) / P_{0} \subseteq$ $\left(I+P_{0}\right) / P_{0}=\operatorname{soc}\left(A / P_{0}\right)$. Let $\pi_{0}$ be a continuous irreducible representation of $A$ on a Banach space $X_{0}$ with $\operatorname{ker} \pi_{0}=P_{0}$. So there exist $c_{0} \in I$, $b_{0} \in J, 0 \neq x_{0} \in X_{0}$ such that $\sigma\left(c_{0}\right) \notin P_{0}, 0 \neq b_{0}+P_{0} \in \operatorname{soc}\left(A / P_{0}\right)$ and $\pi_{0}\left(b_{0}\right) x_{0}=x_{0}$. By Lemma 2.1, $\operatorname{dim}_{\mathbb{C}} \bar{c}_{0}\left(A / P_{0}\right) \bar{b}_{0}=n_{0}<\infty$, where $\bar{x}=x+P_{0}$ for $x \in A$. Then there exist maps $\lambda_{0 i}: A \rightarrow \mathbb{C}, i=1, \ldots, n_{0}$, and $a_{01}, \ldots, a_{0 n_{0}} \in A$ such that $c_{0} a b_{0}-\sum_{i=1}^{n_{0}} \lambda_{0 i}(a) c_{0} a_{0 i} b_{0} \in P_{0}$ for all $a \in A$.

Let $J_{0}=J \cap P_{0}$. We claim that there exists $P_{1} \in \Sigma_{I} \backslash\left\{P_{0}\right\}$ such that $J_{0} \nsubseteq P_{1}$ and $b_{0} \notin P_{1}$. Otherwise, $c_{0} a b_{0}-\sum_{i=1}^{n_{0}} \lambda_{0 i}(a) c_{0} a_{0 i} b_{0} \in\left(J \cap P_{0}\right) \cap$ $\bigcap_{P \in \Sigma_{I} \backslash\left\{P_{0}\right\}} P=0$ for all $a \in A$. This implies $\operatorname{dim}_{\mathbb{C}} c_{0} A b_{0}=n_{0}<\infty$. By Lemma 2.6, $\Phi(\delta) \subseteq P_{0}$, a contradiction. This proves the claim. By Lemma 2.5 ,

$$
0 \neq\left(J_{0}+P_{1}\right) / P_{1} \subseteq\left(I+P_{1}\right) / P_{1}=\operatorname{soc}\left(A / P_{1}\right) .
$$

Let $\pi_{1}$ be a continuous irreducible representation of $A$ on a Banach space $X_{1}$ with ker $\pi_{1}=P_{1}$. So there exist $c_{1} \in I, b_{1} \in J_{0}, 0 \neq x_{1} \in X_{1}$ such that

$$
\sigma\left(c_{1}\right) \notin P_{1}, \quad 0 \neq b_{1}+P_{1} \in \operatorname{soc}\left(A / P_{1}\right), \quad \pi_{1}\left(b_{1}\right) \pi_{1}\left(b_{0}\right) x_{1}=x_{1} .
$$

By Lemma 2.1, $\operatorname{dim}_{\mathbb{C}} \bar{c}_{1}\left(A / P_{1}\right) \bar{b}_{1} \bar{b}_{0}=n_{1}<\infty$. Notice that $\pi_{0}\left(b_{1} b_{0}\right) x_{0}=0$ since $b_{1} \in P_{0}$.

Suppose now that we have primitive ideals $P_{0}, P_{1}, \ldots, P_{k} \in \Sigma_{I}$ and elements $b_{0}, b_{1}, \ldots, b_{k} \in I$ and $c_{1}, \ldots, c_{k} \in I$ such that

- $b_{i} \in J_{i-1}=J \cap P_{0} \cap P_{1} \cap \cdots \cap P_{i-1}$ for all $1 \leq i \leq k$,

- $\operatorname{dim}_{\mathbb{C}} \bar{c}_{i}\left(A / P_{i}\right) \bar{b}_{i} \cdots \bar{b}_{0}=n_{i}<\infty$ for all $1 \leq i \leq k$,

- $\sigma\left(c_{i}\right) \notin P_{i}$ for all $1 \leq i \leq k$.

Further, for each $i \geq 1$, there exist a continuous irreducible representation $\pi_{i}$ of $A$ on a Banach space $X_{i}$ with $\operatorname{ker} \pi_{i}=P_{i}$ and $x_{i} \in X_{i}$ satisfying

$$
\pi_{j}\left(b_{j} \cdots b_{1} b_{0}\right) x_{j} \neq 0 \quad \text { and } \quad \pi_{i}\left(b_{j} \cdots b_{1} b_{0}\right) x_{i}=0 \quad \text { for all } 0 \leq i<j \leq k .
$$

Since $\operatorname{dim}_{\mathbb{C}} \bar{c}_{k}\left(A / P_{k}\right) \bar{b}_{k} \cdots \bar{b}_{1} \bar{b}_{0}=n_{k}$, there exist maps $\lambda_{k i}: A \rightarrow \mathbb{C}, i=$ $1, \ldots, n_{k}$ and $a_{k 1}, \ldots, a_{k n_{k}} \in A$ such that $c_{k} a b_{k} \cdots b_{1} b_{0}-\sum_{i=1}^{n_{k}} \lambda_{k i}(a) c_{k} a_{k i} b_{k}$ $\cdots b_{1} b_{0} \in P_{k}$ for all $a \in A$. Let $J_{k}=J_{k-1} \cap P_{k}=J \cap P_{0} \cap P_{1} \cap \cdots \cap P_{k}$. We 
claim that there exists $P_{k+1} \in \Sigma_{I} \backslash\left\{P_{0}, P_{1}, \ldots, P_{k}\right\}$ such that $J_{k} \nsubseteq P_{k+1}$ and $b_{k} \cdots b_{1} b_{0} \notin P_{k+1}$. Otherwise,

$$
c_{k} a b_{k} \cdots b_{1} b_{0}-\sum_{i=1}^{n_{k}} \lambda_{k i}(a) c_{k} a_{k i} b_{k} \cdots b_{1} b_{0} \in J_{k} \cap\left(\bigcap_{P \in \Sigma_{I} \backslash\left\{P_{0}, P_{1}, \ldots, P_{k}\right\}} P\right)=0
$$

for all $a \in A$. Then we conclude that $\operatorname{dim}_{\mathbb{C}} c_{k} A b_{k} \cdots b_{1} b_{0} \leq n_{k}<\infty$. By Lemma 2.6, $\Phi(\delta) \subseteq P_{k}$, a contradiction. This proves the claim. By Lemma 2.5, $0 \neq\left(J_{k}+P_{k+1}\right) / P_{k+1} \subseteq\left(I+P_{k+1}\right) / P_{k+1}=\operatorname{soc}\left(A / P_{k+1}\right)$. Let $\pi_{k+1}$ be a continuous irreducible representation of $A$ on a Banach space $X_{k+1}$ with ker $\pi_{k+1}=P_{k+1}$. So there exist $c_{k+1} \in I, b_{k+1} \in J_{k}$, $0 \neq x_{k+1} \in X_{k+1}$ such that $\sigma\left(c_{k+1}\right) \notin P_{k+1}, 0 \neq b_{k+1}+P_{k+1} \in \operatorname{soc}\left(A / P_{k+1}\right)$, $\pi_{k+1}\left(b_{k+1}\right) \pi_{k+1}\left(b_{k} \cdots b_{1} b_{0}\right) x_{k+1}=x_{k+1}$. By Lemma 2.1, $\operatorname{dim}_{\mathbb{C}} \bar{c}_{k+1}\left(A / P_{1}\right)$ $\bar{b}_{k+1} \bar{b}_{k} \cdots \bar{b}_{1} \bar{b}_{0}=n_{k+1}<\infty$. Moreover, $\pi_{i}\left(b_{k+1} \cdots b_{1} b_{0}\right) x_{i}=0$ for all $1 \leq$ $i \leq k$ since $b_{k+1} \in P_{0} \cap \cdots \cap P_{k}$.

Proceeding in the same way as above, we may obtain a sequence $\left\{b_{i}\right\}_{i=0}^{\infty}$ in $I$, a sequence $\left\{c_{i}\right\}_{i=1}^{\infty}$ in $I$ and a sequence $\left\{P_{i}\right\}_{i=1}^{\infty}$ of primitive ideals in $\Sigma_{I}$ such that $\sigma\left(c_{n}\right) \notin P_{n}, \pi_{n}\left(b_{n} \cdots b_{1} b_{0}\right) x_{n} \neq 0$ and $\pi_{n}\left(b_{m} \cdots b_{1} b_{0}\right) x_{n}=0$ for all $m>n \geq 1$, where $\pi_{n}$ is a continuous irreducible representation of $A$ on the Banach space $X_{n}$ with ker $\pi_{n}=P_{n}$. In view of Lemma 2.4, $\Phi(\delta) \subseteq P_{i}$ for some $i \geq 1$, a contradiction. This forces $\Sigma_{I}=\emptyset$, as desired. Finally, if $I$ is an essential ideal of $A$ and $\sigma$ is a linear automorphism of $A$, then $\sigma(I)$ is an essential ideal of $A$. In particular, $I \cap \sigma(I)$ is also an essential ideal of $A$. Then from $\Phi(\delta)(I \cap \sigma(I))=0$, it follows that $\Phi(\delta)=0$. The proof is now complete.

Clearly, Corollary 1.2 follows directly from Theorem 1.1. Also, we have

Corollary 3.1. Let $A$ be a semisimple Banach algebra with a linear automorphism $\sigma$ and let $I$ be a closed essential ideal of $A$. Suppose that $\delta: I \rightarrow A$ is a $\sigma$-derivation defined on $I$. Then $\delta$ is continuous.

Recall that an automorphism $\sigma$ of a unital algebra $A$ is called inner if there exists an invertible element $u \in A$ such that $\sigma(a)=u a u^{-1}$ for all $a \in A$. Given any derivation $d$ of $A$ and an invertible element $u \in A$, the map defined by $a \in A \mapsto u d(a)$ is a $\sigma_{u}$-derivation, where $\sigma_{u}: a \in A \mapsto u a u^{-1}$ is an inner automorphism. Obviously, every inner automorphism is continuous. Moreover, if $P$ is a primitive ideal of $A$ and $\sigma$ is inner, then $I \subseteq P$ if and only if $\sigma(I) \subseteq P$. The next result can be regarded as an extension of the corresponding theorem for derivations and is an immediate consequence of Theorem 1.1.

Corollary 3.2. Let $A$ be a semisimple Banach algebra, $\sigma$ an inner automorphism of $A$ and $\delta: I \rightarrow A$ a $\sigma$-derivation, where $I$ is a nonzero ideal of $A$. Then $\Phi(\delta) I=0$. 
A Banach algebra $A$ is called ultraprime if there exists $K>0$ such that $K\|a\|\|b\| \leq\left\|M_{a, b}\right\|$ for all $a, b \in A$, where $M_{a, b}$ denotes the two-sided multiplication operator on $A$ defined by $M_{a, b}(x)=a x b$ for $x \in A$. Obviously, every ultraprime Banach algebra is a prime algebra. By [20, Proposition 2.3], every prime $C^{*}$-algebra is ultraprime and semisimple.

THEOREM 3.3. Let $A$ be an ultraprime Banach algebra with a linear automorphism $\sigma$ and let $I$ be a nonzero ideal of $A$. If $\delta: I \rightarrow A$ is a nonzero closable $\sigma$-derivation, then both $\delta$ and $\sigma$ are continuous.

It is clear that every nonzero ideal in a prime algebra is essential. Applying Theorem 1.1 and 3.3 , we have

COROLlary 3.4. Let $A$ be an ultraprime semisimple Banach algebra with a linear automorphism $\sigma$ and let $I$ be an ideal of A. Then every $\sigma$ derivation defined on $I$ is continuous.

Since every prime $C^{*}$-algebra is ultraprime [20, Proposition 2.3], Corollary 1.3 follows directly from Corollary 3.4. We now turn to the

Proof of Theorem 3.3. For $b \in I$, let $L_{b}: A \rightarrow I$ and $R_{b}: A \rightarrow I$ be the linear operators given by $L_{b}(x)=b x$ and $R_{b}(x)=x b$ for $x \in A$. We claim that the operator $\delta R_{b}: A \rightarrow A$ is continuous. Let $\left\{x_{n}\right\}_{n=1}^{\infty}$ be a sequence in $A$ with

$$
\lim _{n \rightarrow \infty} x_{n}=0 \text { and } \lim _{n \rightarrow \infty} \delta R_{b}\left(x_{n}\right)=\lim _{n \rightarrow \infty} \delta\left(x_{n} b\right)=x .
$$

Since $\delta$ is closable and $\lim _{n \rightarrow \infty} x_{n} b=0, x_{n} b \in I$, we have $x=0$. That is, $\Phi\left(\delta R_{b}\right)=0$. By the closed graph theorem, $\delta R_{b}$ is continuous. Similarly, $\delta L_{b}$ is also continuous.

We claim that $\sigma$ is continuous. Let $\left\{x_{n}\right\}_{n=1}^{\infty}$ be a sequence in $A$ with $\lim _{n \rightarrow \infty} x_{n}=0$ and $\lim _{n \rightarrow \infty} \sigma\left(x_{n}\right)=x$. For $b, c \in I$, since $\delta R_{b c}$ and $\delta R_{b}$ are continuous, we have

$$
\begin{aligned}
0 & =\lim _{n \rightarrow \infty} \delta R_{b c}\left(x_{n}\right)=\lim _{n \rightarrow \infty} \delta\left(x_{n} b c\right)=\lim _{n \rightarrow \infty}\left(\sigma\left(x_{n} b\right) \delta(c)+\delta\left(x_{n} b\right) c\right) \\
& =\lim _{n \rightarrow \infty}\left(\sigma\left(x_{n}\right) \sigma(b) \delta(c)+\delta R_{b}\left(x_{n}\right) c\right)=x \sigma(b) \delta(c) .
\end{aligned}
$$

This implies that $x \sigma(b) \delta(c)=0$ for all $b, c \in I$. Hence $x \sigma(I) \delta(I)=0$. By primeness of $A$ and $\delta \neq 0$, we see that $\delta(I) \neq 0$ and so $x=0$, implying the continuity of $\sigma$.

For $a, b \in I$ and $x \in A$, we have $\delta(a x b)=\sigma(a) \delta(x b)+\delta(a) x b$. That is,

$$
M_{\delta(a), b}(x)=\delta R_{b} L_{a}(x)-L_{\sigma(a)} \delta R_{b}(x) .
$$

Note that $\delta R_{b} L_{a}$ and $L_{\sigma(a)} \delta R_{b}$ are continuous. Thus $\left\|M_{\delta(a), b}\right\| \leq\left\|\delta R_{b} L_{a}\right\|+\left\|L_{\sigma(a)} \delta R_{b}\right\| \leq\|a\|(1+\|\sigma\|)\left\|\delta R_{b}\right\| \quad$ for all $a, b \in I$. By assumption, there exists $K>0$ such that $K\|\delta(a)\|\|b\| \leq\left\|M_{\delta(a), b}\right\|$ for all $a, b \in I$. So $\|\delta(a)\| \leq K^{\prime}\|a\|$ for some $K^{\prime}>0$. This proves the theorem. 
Before proving our last result, we refer the reader to [2, Chapter 2] for the notion of the symmetric algebra of quotients of a semisimple algebra. Theorem 1.4 is an immediate consequence of Theorem 3.5 below.

Theorem 3.5. Let $A$ be a prime semisimple Banach algebra and let $Q$ be the symmetric algebra of quotients of $A$. Suppose that $Q$ contains a nontrivial idempotent and $\delta: A \rightarrow A$ is a linear map. If $\sigma(x) \delta(y)+\delta(x) y=0$ for all $x, y \in A$ with $x y=0$, where $\sigma$ is a linear automorphism of $A$, then there exists a nonzero ideal $J$ of $A$ such that $\delta: J \rightarrow A$ is closable. In addition, if $e A \cup A e \subseteq A$ for some nontrivial idempotent $e \in Q$, then $\delta$ is continuous.

Proof. In view of [18, Theorem 1.1], there exist $a, b \in Q$, a nonzero ideal $J$ of $A$ and a $\sigma$-derivation $d: A \rightarrow Q$ such that $\delta(x)=d(x)+\sigma(x) b=$ $d(x)+a x$ for all $x \in J$. Moreover, $J=A$ if $e A \cup A e \subseteq A$ for some nontrivial idempotent $e \in Q$. Choose a nonzero ideal $K$ of $A$ such that $K \subseteq J$ and $b K \cup K b \subseteq A$. Set $I=K \cap \sigma^{-1}(K)$. Then $K$ is a nonzero ideal of $A$ such that $I \subseteq J$ and $\sigma(I) b \cup b I \subseteq A$. Since $d(x)=\delta(x)-\sigma(x) b$ for $x \in I$, we see that $d(I) \subseteq A$.

Let $x \in J$ and $y \in I$. Then $\delta(x) y=d(x) y+\sigma(x) b y=d(x y)-\sigma(x) d(y)+$ $\sigma(x)(b y)$. That is, $R_{y} \delta(x)=d R_{y}(x)-R_{d(y)} \sigma(x)+R_{b y} \sigma(x)$. By Theorem 1.1, $d: I \rightarrow A$ is closable. By the same proof given in Theorem 3.3, $d R_{y}: A \rightarrow$ $A$ is continuous. Let $\left\{x_{n}\right\}_{n=1}^{\infty}$ be a sequence in $J, \lim _{n \rightarrow \infty} x_{n}=0$ and $\lim _{n \rightarrow \infty} \delta\left(x_{n}\right)=x \in \Phi(\delta)$. Since $\sigma$ is continuous [23, Corollary 6.12], it is easy to see that $0=\lim _{n \rightarrow \infty} R_{y} \delta\left(x_{n}\right)=x y$. Hence $x I=0$ and then $x A I=0$. By primeness of $A, x=0$. Thus $\Phi(\delta)=0$. This proves the theorem.

Acknowledgements. The authors are thankful to the referee for the very thorough reading of the paper and for valuable suggestions.

\section{References}

[1] Z. Abdelali, On $\Phi$-derivation in Banach algebras, Comm. Algebra 34 (2006), 24372452 .

[2] P. Ara and M. Mathieu, Local Multipliers of $C^{*}$-algebras, Springer, London, 2003.

[3] M. I. Berenguer and A. R. Villena, Continuity of Lie mappings of the skew elements of Banach algebras with involution, Proc. Amer. Math. Soc. 126 (1998), 2717-2720.

[4] - - - Continuity of Lie derivations on Banach algebras, Proc. Edinburgh Math. Soc. 41 (1998), 625-630.

[5] M. Brešar, On automorphisms of Banach algebras, Arch. Math. (Basel) 78 (2002), 297-302.

[6] M. Brešar, A. Fošner and M. Fošner, A Kleinecke-Shirokov type condition with Jordan automorphisms, Studia Math. 147 (2001), 237-242.

[7] M. Brešar and P. Šemrl, Finite rank elements in semisimple Banach algebras, ibid. 128 (1998), 287-298. 
[8] M. Brešar and P. Šemrl, Commutativity preserving linear maps on central simple algebras, J. Algebra 284 (2005), 102-110.

[9] M. Brešar and A. R. Villena, The noncommutative Singer-Wermer conjecture and $\phi$-derivations, J. London Math. Soc. 66 (2002), 710-720.

[10] M. A. Chebotar, W.-F. Ke and P.-H. Lee, Maps characterized by actions on zero products, Pacific J. Math. 216 (2004), 217-228.

[11] M. A. Chebotar, W.-F. Ke, P.-H. Lee and N.-C. Wong, Mappings preserving zero products, Studia Math. 155 (2003), 77-94.

[12] C.-L. Chuang and C.-K. Liu, Extended Jacobson density theorem for rings with skew derivations, Comm. Algebra 35 (2007), 1391-1413.

[13] - - -, Skew derivations mapping into the socle, manuscript.

[14] W. Jing, S. Lu and P. Li, Characterizations of derivations on some operator algebras, Bull. Austral. Math. Soc. 66 (2002), 227-232.

[15] B. E. Johnson and A. M. Sinclair, Continuity of derivations and a problem of Kaplansky, Amer. J. Math. 90 (1968), 1067-1073.

[16] I. Kaplansky, Functional Analysis. Some Aspects of Analysis and Probability, Surveys Appl. Math. 4, Wiley, New York, and Chapman \& Hall, London, 1958.

[17] —, Derivations of Banach algebras, in: Seminars on Analytic Functions, Vol. II, Institute for Advanced Study, Princeton, 1958, 254-258.

[18] T.-K. Lee, Generalized skew derivations characterized by acting on zero products, Pacific J. Math. 216 (2004), 293-301.

[19] T.-K. Lee and C.-K. Liu, Spectrally bounded $\phi$-derivations on Banach algebras, Proc. Amer. Math. Soc. 133 (2005), 1427-1435.

[20] M. Mathieu, Elementary operators on prime $C^{*}$-algebras I, Math. Ann. 284 (1989), 223-244.

[21] M. Mirzavaziri and M. S. Moslehian, Automatic continuity of $\sigma$-derivations on $C^{*}$ algebras, Proc. Amer. Math. Soc. 134 (2006), 3319-3327.

[22] S. Sakai, On a conjecture of Kaplansky, Tôhoku Math. J. 12 (1960), 31-33.

[23] A. M. Sinclair, Automatic Continuity of Linear Operators, London Math. Soc. Lecture Note Ser. 21, Cambridge Univ. Press, Cambridge, 1976.

[24] —, Jordan homomorphisms and derivations on semisimple Banach algebras, Proc. Amer. Math. Soc. 24 (1970), 209-214.

[25] M. P. Thomas, Primitive ideals and derivations on noncommutative Banach algebras, Pacific J. Math. 159 (1993), 139-152.

[26] A. R. Villena, Derivations on Jordan-Banach algebras, Studia Math. 118 (1996), 205-229.

[27] —, Essentially defined derivations on semisimple Banach algebras, Proc. Edinburgh Math. Soc. 40 (1997), 175-179.

[28] W. J. Wong, Maps on simple algebras preserving zero products. I. The associative case, Pacific J. Math. 89 (1980), 229-247.

Department of Mathematics

National Taiwan University

Taipei 106, Taiwan

E-mail: tklee@math.ntu.edu.tw
Department of Mathematics National Changhua University of Education

Changhua 500, Taiwan E-mail: ckliu@cc.ncue.edu.tw 